\title{
True Colors: The Power of Spirit in Caring for and Rehabilitating the Aging Body
}

\author{
Sophia 0JM Dijkstra* \\ Aging Gracefully, San Jose, USA
}

*Corresponding author: Sophia OJM Dijkstra, Aging Gracefully, San Jose, CA 95118, Tel: 408448 4459/408 694 2919; Email: fieke@comcast.net

Submission: 侮January 18, 2018; Published: 海J January 22, 2018

\section{Opinion}

The content of this article is not based on years of scientific research, objective data collection, statistical comparisons or outcome measures. It has no references or a lengthy bibliography. Instead, it is an account of almost forty years of providing geriatric rehabilitation to seniors in long-term care facilities. It is a tale of daily interaction and connection with ordinary people in extraordinary circumstances, where a physical impairment affects the body, overwhelms the mind and subdues the spirit.

My outcomes are smiles and happy tears, my case studies are elderly people working hard to regain physical function, often against incredible odds. As the mind struggles to overcome impatience and frustration, the spirit faces a sense of loss and inevitable existential questions. Clinicians-including physicians, nurses, physical therapists, etc. -are trained to place the primary focus on the medical, most likely physical problems of our patients. We are treating them to fix or cure physical problems that impact physical function. We set physically focused goals, often without asking our patients what their goals are. Over the years I have discovered that successful physical intervention can only be accomplished when we move away from this strictly "medical model" and turn toward the "human model". This means that the focus has to shift from fixing and curing to healing while offering less treatment and more care. A patient must be approached as a person with a mind and a soul, not only a body.

Each person is unique having his or her own individual colors, expressed in physical features, personality traits, gifts and talents, acquired knowledge and skills, and a lifetime of experiences. The colors of body and mind will change as life progresses, but the colors of the spirit-the soul, our essential, deepest self - do not change. They are our true colors. Changes in body and mind caused by injury, disease, disability, dementia or mental illness can manifest in pain, discomfort, fear, anxiety, frustration or even depression. Someone's true colors may be temporarily hidden or covered by these conditions. Dementia, for example, causes the colors of the mind and body to slowly fade away the true colors, but does never erase the true colors of the soul. As clinicians we need to be aware of this process to ensure an appropriate approach to our interventions. "Difficult" patients are not really difficult if the underlying adverse conditions are identified, and we understand and practice effective ways of communicating and connecting with them.

As a geriatric physical therapist, I recognize that seniors need a longer recovery from illness than do younger people because their bounce back is less flexible. Many are afflicted by permanent, progressive or degenerative disabilities with multiple co-morbidities. Socially they often have a limited support system and they are grappling with existential questions, especially at more advanced ages since they have become closer to the reality of mortality.

As long as our bodies are healthy and we can use them for all the things we have to do and like to do, our predominant experience is that we "have" a body. If our bodies stop functioning, we have a different experience, namely that we "are" our bodies and these bodies become a prison, deeply affecting our spirits. As Carter Catlett Williams states: The interconnectedness of body and spirit that exists throughout life's journey becomes achingly direct and clear in old age in the presence of chronic disease, many personal losses, and increasing dependency. Body and spirit are then enfolded, one upon another, more closely, inextricably, than at any other time of life, and the skill and understanding-or the lack thereof- with which these body-spirit needs are met either strengthen or break down the spirit. If the spirit breaks, life's savor is lost, though life continues. Conversely, as surely as the body is eased, the spirit is lightened and lifted up."

The role of the caregiver or clinician is to resurrect the patient's true colors, to make a person whole again. As a physical therapist I use a safety belt that I wrap around a person's waist to assist with transfers, gait and balance training. The function of the belt is to provide physical safety. I tell my patients that, whenever the belt is around their waists, they will have my undivided attention. It is their time with me and so the belt is not only a tool for physical safety but it becomes a symbol of emotional and spiritual security. During the therapy session people take the opportunity to talk, to share, to express their feelings and emotions. And slowly their true colors shine through and they show their real selves again. 
The belt has five colored stripes, and over the years it has taken on the hopeful symbolism of a rainbow, reminding my patients that through the clouds of daily struggles the sun always comes shining through. Each color in my rainbow belt represents an aspect of my interaction with the patient beyond the pure clinical treatment, (guidance, reassurance, affirmation, connection, encouragement). Each color also represents my approach (gentleness, respect, attention, compassion, empathy). And last but not least, each color represents a coping tool (gratitude, resilience, attitude, courage, empowerment). The five colors represent the word GRACE (Table 1).

Table 1

\begin{tabular}{|c|c|c|}
\hline Interaction & Approach & Coping Tool \\
\hline Guidance & Gentleness & Gratitude \\
\hline Reassurance & Respect & Resilience \\
\hline Affirmation & Attention & Attitude \\
\hline Connection & Compassion & Courage \\
\hline Encouragement & Empathy & Empowerment \\
\hline
\end{tabular}

The belt has two sides, one with the five colors of an individual: physical features, personality traits, talents and gifts, acquired knowledge and skills and a lifetime of experience (internal grace) and one with the five colors of grace as shown in the grace grid above (external grace). In my work I never talk with my patients about the different components of my rainbow. Instead, I use all the colors of my grace grid to guide a person through the rehabilitation process. I reassure and encourage while establishing a strong connection. Once I feel that my relationship with the patient is based on trust, the door opens to an opportunity to affirm the patient as a person. Geriatric rehabilitation can only be successful when patients are treated with gentleness, respect, true attention, compassion and empathy. During my sessions I put a challenging situation in perspective by suggesting reasons to be grateful, I insist to never give up, to keep a positive attitude, to be courageous. In the end, patients feel empowered to heal and to become the best person they can be.

Not every clinician uses a safety belt, but as Maya Angelou reminds us" Each one of us has the possibility, probability and privilege to be a rainbow in someone's cloud". My grace grid has turned multitudes of difficult and often negative situations into positive experiences with positive results for the elderly going through geriatric rehabilitation. Many diseases and conditions affecting seniors cannot be cured. However, once the healing process is set in motion and quality of life becomes the focus, the patient becomes a person again, living perhaps a restricted life, but meaningful one. 